\title{
Sense of coherence in family caregivers of people living with dementia: a mixed- methods psychometric evaluation
}

\author{
Jacki Stansfeld ${ }^{1,2^{*}}$ (D, Martin Orrell ${ }^{3}$, Myrra Vernooij-Dassen ${ }^{4}$ and Jennifer Wenborn ${ }^{1,2}$
}

\begin{abstract}
Background: Family caregivers of people living with dementia can experience feelings of burden and stress but the concept of sense of coherence has been identified as an important protective trait against the negative impact of caregiving. Despite this, there has been no psychometric evaluation of the Sense of Coherence scale-13 with this population. Therefore, a psychometric evaluation was conducted using a mixed-methods approach.

Method: Five hundred and eighty-three caregivers of people living with dementia participated in the study. We examined the feasibility, internal consistency, construct validity, floor and ceiling effects, concurrent validity and face validity of the Sense of Coherence scale-13.

Results: The Sense of Coherence scale-13 demonstrated adequate internal consistency. Sense of coherence was positively related to resilience, sense of competence and health related quality of life, demonstrating good concurrent validity. However, the face validity of the scale was assessed as poor.

Conclusion: The sense of coherence scale performed well under psychometric evaluation however guidance for caregivers should be examined and revised to reflect feedback from caregivers who completed this study, which could lead to improved face validity for this scale.
\end{abstract}

Trial registration number: ISRCTN10748953. Registered 18th September 2014.

Keywords: Dementia, Alzheimer's', Caregiver, Measurement, Psychometrics, Validity, Reliability, Sense of coherence

\section{Background}

By 2050, it is estimated that there will be over 131 million people with dementia worldwide [1], of whom the majority will be cared for by friends or relatives in the community [2]. Over time, family caregivers may experience feelings of burden and stress [3-5]. The negative impact of caregiving can lead to poor outcomes in caregivers, such as depression, anxiety and physical health problems $[6,7]$. Protective psychological factors can reduce the negative impact of caregiving. One such protective factor is sense of coherence. The concept of sense of coherence emerged from Antonovsky's (1979) salutogenic theory [8], which outlines the unique way in which each person views

\footnotetext{
* Correspondence: j.stansfeld@ucl.ac.uk

'Division of Psychiatry, University College London, Maple House, 149

Tottenham Court Road, London W1T 7BN, UK

${ }^{2}$ Research and Development, North East London NHS Foundation Trust,

Goodmayes Hospital, Ilford IG3 8XJ, UK

Full list of author information is available at the end of the article
}

the world and their individual circumstances as comprehensible, manageable and meaningful. This theory focuses on personal strengths as determinants for quality of life and positive wellbeing [9]. Sense of coherence has increasingly been viewed as an important concept within research with family caregivers of people living with dementia. In this population, sense of coherence has been shown to predict health related quality of life [10] and burden [11] and has been related to the ability to cope with the caregiving role [12].

A number of scales have been developed to measure sense of coherence. The original, 29-item sense of coherence scale was reduced to a 13-item measure, the sense of coherence scale-13 items (SOC-13) $[9,13]$, which is considered a suitable length for research and clinical practice [14]. There are a number of empirical studies incorporating measures of sense of coherence in the dementia family caregiver population (e.g. $[11,15,16])$,

(c) The Author(s). 2019 Open Access This article is distributed under the terms of the Creative Commons Attribution 4.0 International License (http://creativecommons.org/licenses/by/4.0/), which permits unrestricted use, distribution, and reproduction in any medium, provided you give appropriate credit to the original author(s) and the source, provide a link to the Creative Commons license, and indicate if changes were made. The Creative Commons Public Domain Dedication waiver (http://creativecommons.org/publicdomain/zero/1.0/) applies to the data made available in this article, unless otherwise stated. 
however, to our knowledge; there is currently no psychometric evaluation of the sense of coherence scale with family caregivers of people living with dementia. Sense of coherence is important for understanding how to maintain wellbeing and has the potential to be incorporated as an outcome measure in clinical interventions. Therefore, it is important to investigate whether a short sense of coherence measure can be a valid and useful measure for this population.

\section{Aim}

The aim of this research was to examine the psychometric properties (feasibility, subscales, internal consistency, floor and ceiling effects, construct validity and face validity) of the SOC-13 in a population of family caregivers of people with dementia.

\section{Method}

\section{Design}

This was a cross-sectional, mixed-methods study. A mixed-methods design was chosen in order to examine both the psychometric properties of the scale but the caregiver's experience of completing the scale. The quantitative enquiry can therefore be combined with the rich insights of the qualitative data. All participants were recruited within a period of 6 months. Respondents were contacted through either the Join Dementia Research (JDR) database, an online database of people with dementia and their family caregivers who have registered their interest in taking part in UK-based research or relevant charitable organisations for older people, people with dementia and their family caregivers. All participants were asked to read an information sheet before participating and gave informed consent to take part in the study.

\section{Participants}

Caregivers who expressed an interest in the study following advertisements or mail-outs through the JDR or charitable organisations were emailed or contacted by phone with a link to the online version of the survey and offered a paper copy with a freepost envelope for them to return the completed survey if they preferred. To participate in the research, participants had to be aged 18 or over, currently caring for a person living with dementia in the community and able to read and communicate in English. Participants were excluded from the study if the person with dementia they were caring for lived in residential care.

\section{Measures}

There were two sections to the survey. The first asked demographic questions about the family caregiver and the person with dementia, such as gender, age, living situation, ethnicity and marital status. The second section included standardised measures as outlined below.

\section{Sense of coherence}

The Sense of Coherence scale-13 [13] is a 13-item scale adapted from a longer 29-item scale [9]. This scale consists of three subscales: meaningfulness (four items), comprehensibility (five items) and manageability (four items). Each item contains 7 response options, which change between questions. Five variables (items 1, 2, 3, 7 and 10) are negatively worded and are therefore reverse coded when summing the items to reach an item total. A higher score represents a higher sense of coherence. Reliability estimates for this scale range from $\alpha=0.70-0.92$ [14].

\section{Resilience}

The Resilience Scale-14 (RS-14) [17] is a 14-item scale used to measure levels of resilience. Items are scored between 1 (strongly disagree) and 6 (strongly agree), with possible total scores ranging from 14 to 84 . The higher the score, the higher the level of resilience. The RS-14 has demonstrated good levels of reliability with Cronbach's $\alpha$ scores ranging between 0.82 and $0.94[18,19]$.

\section{Sense of competence}

The Short Sense of Competence Questionnaire (SSCQ) [20] is a 7-item measure derived from a longer, 27-item Sense of Competence measure. Scores can range from 7 to 35 and items are scored on a 5-point Likert scale from 1 (agree very strongly) to 5 (disagree very strongly), with a higher score indicating a higher sense of competence. The reliability for the scale is good, with Cronbach's $\alpha$ scores at 0.76 [20].

\section{Self-efficacy}

The Self-efficacy for Managing Dementia Scale (SEMD) [21] is a 10-item scale that measures the level of self-efficacy caregivers experience for managing the task of caregiving. Possible scores range from 10 to 100, with higher scores indicating higher self-efficacy. Responses are arranged on a likert scale from 1 (not at all certain) to 10 (very certain). The reliability for this scale is good $(\alpha=0.77)[21]$.

\section{Health related quality of life}

The EQ-5D-5 L [20] provides a simple descriptive profile and a single index value for health status, which can be used to evaluate quality of life. Higher scores indicate higher health related quality of life. Cronbach's alpha has been reported at 0.85 [22].

\section{Face validity}

Five open text-box questions were included directly after the respondents completed the sense of coherence scale, 
in order to assess face validity. These questions were preceded by an explanation of the sense of coherence scale and what the scale intended to measure and were as follows:

1. Did you find any of the 13 questions difficult to understand?

2. Is there anything you would add to the 13 questions above?

3. Is there anything you would change in the 13 questions above?

4. Is there anything you would remove from the 13 questions above?

5. Do you have any other comments about the 13 questions above?

\section{Data analysis}

Data collected using the online SurveyMonkey format was downloaded from to the IBM SPSS Statistics version 25 [23] and entered manually from the paper surveys. Data was screened for any inconsistencies or errors in data entry. Qualitative data were downloaded or entered separately onto an excel spreadsheet and analysed using inductive thematic analysis [24] by two researchers (JS \& JW). The quantitative and the qualitative data was then utilised in tandem to understand the psychometric properties and acceptability of the scale to caregivers.

The feasibility of the scale was calculated by examining the response rate of the scale and an investigation of missing values per item. Internal consistency was examined using Cronbach's $\alpha$, which was calculated for the scale as a whole and for each of the subscales. Cronbach's $\alpha$ is considered acceptable if it falls between 0.7-0.9.

A confirmatory factor analysis (CFA) was conducted in order to explore whether the SOC-13 adequately measured the three domains (comprehensibility, manageability and meaningfulness) as established in the original measure. Confirmatory factor indices values of $>.90$, SRMR values of $<.08$ and RMSEA values of between .06 and .08 were considered acceptable.

Floor and ceiling effects are considered important in psychometric evaluation because if these effects are present, it limits the scale's ability to detect a change in the construct. Floor and ceiling effects were investigated by generating histograms and calculating frequencies to examine the proportion of scores that fell within the highest and lowest possible scores on the scales. Floor effects were considered to be present if above $15 \%$ of respondents had the lowest possible scores. Ceiling effects were considered to be present if $15 \%$ of respondents had the highest possible scores on the scale as in previous psychometric research [25].

Concurrent validity was calculated using Pearson's correlation coefficients to examine the relationship between sense of coherence and the other measures, based on previous research and an underlying theory of sense of coherence. Relationships between the variables were considered low if $\mathrm{r}<0.29$, moderate if $\mathrm{r}<0.3$ to $\mathrm{r}<0.40$ and high if $r<0.41$ to $r<1$ (Field, 2009) [26]. Hypotheses in terms of the relationships between variables were specified a priori:

1. Sense of coherence will be positively correlated with caregiver's resilience. It is plausible that the way in which a person views their life may impact their resilience to deal with stressful or difficult circumstances. This association has been found in previous literature [27, 28].

2. Sense of coherence will be positively correlated with sense of competence and self-efficacy for managing dementia, as theoretically, an ability to find meaning and understanding in the caregiving situation (sense of coherence) could lead to a higher perceived level of competence and higher self-efficacy to deal with the task of caregiving. Self-efficacy and sense of competence were both assessed in relation to sense of coherence as despite a conceptual overlap, they measure distinct concepts.

3. Sense of coherence will be positively associated with health-related quality of life. This relationship has been demonstrated in previous literature [10].

\section{Results}

583 caregivers of people living with dementia completed the survey. Of these, $516(89 \%)$ participants opted to complete it online and 67 (11\%) of participants completed a paper version. There were no significant differences between responses on the online and paper questionnaire in terms of skewness and kurtosis of the scales, scores on the standardised measures and most of the key demographics. There was however, a difference between the ages of family caregivers who completed the online and paper questionnaire $(\mathrm{t}=-6.7, p=.00)$. Caregivers who completed the online questionnaire were significantly younger (mean age $=58.3$ ) than those who completed the paper version (mean age $=69$ ).

\section{Demographics of the family caregivers and people with dementia}

The mean age of caregivers taking part in the survey was 59.5 years, with a range of $18-89$ years. The majority of family caregivers were female $(80.3 \%)$, white British or Irish (94.2\%) and adult child caregivers (58.9\%). Over half of the caregivers were married (68.9\%), with $71.3 \%$ having completed further education. The people with dementia that were cared for had a mean age of 80 years old, with a range of 39-99. The majority were female $(63.4 \%)$ and half were married (50.3\%). Almost all were 
Table 1 Family caregiver and person with dementia descriptive demographics in the whole sample $(n=583)$

\begin{tabular}{|c|c|}
\hline Family caregiver demographics & Total \\
\hline \multicolumn{2}{|l|}{ Gender $n(\%)$} \\
\hline Female & $468(80.3)$ \\
\hline Male & $114(19.7)$ \\
\hline Age $M(S D)$ & $59.5(12.3)$ \\
\hline Range & $18-89$ \\
\hline \multicolumn{2}{|l|}{ Marital status $n(\%)$} \\
\hline Single & $81(14.1)$ \\
\hline Married & $397(68.9)$ \\
\hline Separated & $13(2.3)$ \\
\hline Divorced & $42(7.3)$ \\
\hline Widow/widower & $13(2.3)$ \\
\hline Other & $28(4.9)$ \\
\hline Prefer not to say & $2(0.3)$ \\
\hline \multicolumn{2}{|l|}{ Ethnicity $n(\%)$} \\
\hline White British/ Irish & $543(94.2)$ \\
\hline Mixed British & $4(0.7)$ \\
\hline Indian/British Indian & $4(0.7)$ \\
\hline Black Caribbean/African & $4(0.7)$ \\
\hline Other & $19(3.7)$ \\
\hline \multicolumn{2}{|c|}{ Highest completed level of education $n(\%)$} \\
\hline Primary education or less & $3(0.5)$ \\
\hline Secondary education & $136(23.7)$ \\
\hline Further education & $409(71.3)$ \\
\hline Other general education & $20(3.5)$ \\
\hline Prefer not to say & $6(1)$ \\
\hline \multicolumn{2}{|c|}{ Relationship to person with Dementia $n(\%)$} \\
\hline Spouse/partner & $173(30.1)$ \\
\hline Son/daughter & $338(58.9)$ \\
\hline Other & $63(11)$ \\
\hline \multicolumn{2}{|l|}{ Cohabiting $n(\%)$} \\
\hline Yes & $245(42.7)$ \\
\hline No & $329(57.3)$ \\
\hline \multicolumn{2}{|c|}{ Receiving additional support $\mathrm{n}(\%)(n=572)$} \\
\hline Yes & $390(68.2)$ \\
\hline No & $182(31.8)$ \\
\hline \multicolumn{2}{|c|}{ Person with dementia demographics } \\
\hline \multicolumn{2}{|l|}{ Gender $n(\%)$} \\
\hline Female & $369(63.4)$ \\
\hline Male & $213(36.6)$ \\
\hline Age $M(S D)$ & $80(9.5)$ \\
\hline Range & $39-99$ \\
\hline \multicolumn{2}{|l|}{ Marital status $n(\%)$} \\
\hline Single & $17(3)$ \\
\hline
\end{tabular}

Table 1 Family caregiver and person with dementia descriptive demographics in the whole sample $(n=583)$ (Continued)

\begin{tabular}{ll} 
Married & $287(50.3)$ \\
Separated & $10(1.8)$ \\
Divorced & $25(4.4)$ \\
Widow/widower & $227(39.8)$ \\
Other & $5(0.9)$ \\
Prefer not to say & - \\
Ethnicity $n$ (\%) & \\
White British/ Irish & $541(95.2)$ \\
Mixed British & - \\
Indian/British Indian & $5(0.9)$ \\
Black Caribbean/African & $5(0.9)$ \\
Other & $4(0.7)$ \\
Highest completed level of education $n(\%)$ & \\
Primary education or less & $30(5.3)$ \\
Secondary education & $313(55.2)$ \\
Further education & $163(27.9)$ \\
Other general education & $28(4.9)$ \\
Prefer not to say/not known & $33(5.9)$ \\
Dementia diagnosis $n$ (\%) & \\
Alzheimer's Disease & $288(50.5)$ \\
Vascular & $108(18.9)$ \\
Dementia with Lewy & $19(3.3)$ \\
Fodies & $22(3.9)$ \\
& $133(23.3)$ \\
\hline
\end{tabular}

white British or Irish (95.2\%) and the majority had completed secondary education (55.2\%). Half of the people with dementia had an Alzheimer's disease diagnosis (50.5\%). Demographics of the family caregivers and people with dementia is further detailed in Table 1. Where totals do not add to 583, it indicates that some carers did not leave responses for the demographic questions.

\section{The profile of sense of coherence}

Generally, the sense of coherence scores had a normal distribution but demonstrated a positive skew in the entire population, indicating the tendency of family caregivers of people living with dementia to report higher levels of sense of coherence. The mean sense of coherence score was 60.2 and the standard deviation of scores was 14 . There was a total range from 17 to 90 across this population.

\section{Feasibility}

Some participants opted out of completing the full survey and therefore there was a completed sense of coherence scale received from 547 family caregivers of people living with dementia. However, there were no missing values or 
items in any of the returned sense of coherence scales, indicating good feasibility in the measure itself.

\section{Internal consistency}

Overall, the SOC-13 demonstrated good internal consistency $(\alpha=0.883)$. The comprehensibility subscale had good internal consistency $(\alpha=0.76)$ and the range of item-total correlations ranged from $r=0.176$ to 0.608 . In the manageability subscale, Cronbach's $\alpha$ was good, at $\alpha=$ 0.705 and the range of item-total correlations ranged from $r=0.207-0.667$. In the meaningfulness subscale there was good internal consistency $(\alpha=0.72)$ and the range of item-total correlations ranged between $r=0.39-0.63$. All of the item-total correlations were $>0.3$. The smallest item-total correlation was for item $3(\mathrm{r}=0.39, p<.001)$.

\section{Construct validity}

A CFA indicated that the items did not confirm the originally proposed three factor structure. Syntax was entered into MPlus to specify the three latent factors, 'meaningfulness', 'manageability' and 'comprehensibility'. These latent factors were allowed to correlate freely. The results indicated that the proposed model was not an adequate fit, with indices falling below or above acceptable limits (Table 2). However, all factor loadings were significant and ranged from 0.419-2.124. There was a small amount of covariance between the subscales.

\section{Floor and ceiling effects}

Floor and ceiling effects were not present in any of the items. None of the participants scored the maximum or minimum score on this scale.

\section{Concurrent validity}

The hypotheses for concurrent validity were confirmed. Sense of coherence was strongly and positively correlated with resilience $(r=0.56, p<.001)$. Sense of coherence was also moderately and positively correlated with sense of competence $(\mathrm{r}=0.42, p<.001)$, and self-efficacy for managing dementia $(\mathrm{r}=0.46, p<.001)$. These results indicate that as sense of coherence increases, so does resilience, self-efficacy and sense of competence. Sense of coherence was also moderately correlated with health-related quality of life $(\mathrm{r}=-0.38, p<.001)$. As health-related quality of life is reverse scored, a negative

Table 2 CFA validation of the original 3 factor structure of the SOC-13

\begin{tabular}{llllcl}
\hline & $\times 2$ & df & CFI & RMSEA & SRMR \\
\hline 3 Factors & $424.529^{*}$ & 62 & 0.867 & 0.103 & 0.056 \\
\hline
\end{tabular}

$\times 2=$ Chi-Square goodness of fit; $d f$ degrees of freedom, RMSEA Root Mean Square Error of Approximation, SRMR Standardised Square Root Mean Residual. *Statistically significant at $p<.001$ correlation indicates that as sense of coherence increases, so does health related quality of life (Table 3).

\section{Face validity}

A total of 462 caregivers gave at least one comment in the open-text boxes provided. Thematic analysis identified the following themes related to the (1) question content, (2) scoring and (3) relevance to caregiver role. Each of the themes is discussed below and illustrated with data excerpts.

\section{Question content}

Respondents felt that the questions made them think about their situation and this was considered a positive thing, as it gave them a chance to consider their feelings and reflect upon their situation.

'Made me realise how competence and capable I am and how much I love my husband at this stage in his life...our lives' [female, aged 72]

However, some caregivers reported that the questions were vague and did not give enough detail as to enable an answer or to orientate them.

'Some [questions] are ambiguous, some too vague e.g.: "do you have the feeling that you're being treated unfairly?" By whom? My relative? My family? Society? Life in general?' [male, aged 57]

Caregivers noted problems with specific questions, particularly question 10, 'Many people - even those with a strong character - sometimes feel like sad sacks (losers) in certain situations. How often have you felt this way in the past?' Generally, caregivers took offence at the word 'loser' and did not recognise the term 'sad sacks' as this is not a common term used in the UK.

\section{'Losers is not a very helpful phrase' [Male, aged 85]}

In addition, caregivers reported confusion with question 12, "How often do you have the feeling that there's little meaning in the things you do in daily life?" They felt that meaning was a very ambiguous term and not easy to categorise.

'The question about 'meaning' is an odd one. I know my life has little meaning in the grander scheme, but that doesn't mean I'm not happy in my little bit.'

[Female, aged 49]

\section{Scoring}

The nature of the scoring created problems for respondents in terms of choosing the difference between scores 
Table 3 Pearson's correlations for sense of coherence

\begin{tabular}{lllll}
\hline & Sense of competence & Self-efficacy & Health-related quality of life & Sense of coherence \\
\hline Resilience & $.254^{* * *}$ & $.455^{* * *}$ & $.301^{* * *}$ & $.561^{* * *}$ \\
Sense of competence & - & $.354^{* * *}$ & $.275^{* * *}$ & $.223^{* * *}$ \\
Self-efficacy & - & - & - & $.419^{* * *}$ \\
Health-related quality of life & - & - & $-.377^{* * * *}$ \\
\hline
\end{tabular}

${ }^{*} p<.05,{ }^{* *} p<.01,{ }^{* * *}<p<.001$

on the scale and quantifying scores between 1 and 7. As the response options changed with each question, caregivers found this confusing and sometimes difficult to select the most relevant number.

'They aren't easy to quantify which made them tricky to answer' [male, aged 57]

\section{Relevance to caregiver role}

Some of the response options were difficult for the caregivers to answer as they felt that there was no constant when living with someone with dementia. Life was not predictable and may change from one day to the next.

'[The questions are] not [difficult] to understand just difficult to answer as there is not a constant in...living with someone with dementia' [Female, aged 53]

Dementia is a degenerative condition and therefore caregivers felt that their response would change as their friend/relative's dementia progressed and became more severe.

'This only applies to now, things may change as my husband's condition deteriorates' [Female, aged 75]

Caregivers also felt the instructions for the sense of coherence scale and the questions were not specific enough. Caregivers reported the need for distinction as to whether the questions were aiming to reflect their life in general or their caregiving role specifically in relation to the person with dementia that they support.

I found it hard to disentangle responses in relation to how I feel about coping with and supporting my mum with Alzheimer's and the other part of my life.'

[Female, aged 61]

\section{Discussion}

The strength of this study is that it provides a psychometric evaluation using a mixed methods approach, of a well-used and popular scale in a new population. The sense of coherence scale is commonly used in empirical research and has been used in at least 33 languages, with many different clinical populations [14]. This scale has been commonly used with family caregivers of people living with dementia but has not been validated in this population. Overall, good psychometric properties were demonstrated for using this scale in this population. In previous research, Cronbach's $\alpha$ scores ranged from 0.70 to 0.92 across 127 studies [14] and good scores were also found within this psychometric evaluation $(\alpha=0.88)$. Generally, the results from this psychometric evaluation reflected those reported in a previous systematic review of the reliability and validity of the sense of coherence scale in the general population [14]. Feasibility was also found to be high as there were no missing items or caregivers failing to complete the whole scale once they had started. This demonstrates that the scale was acceptable to caregivers in a self-report format. As the scale is normally used in an interviewer-administered format, it is promising to see that a self-report format did not affect completion rates. The scale had good internal consistency, with good Cronbach's alpha scores for the entire scale and the subscales, which were similar for scores in the general population reported in a previous systematic review [14].

There were no floor and ceiling effects present in the scale as a whole or in any of the subscales within the population of family caregivers of people living with dementia. This indicates the suitability of the scale to be used in interventional research.

In terms of concurrent validity, all of the hypotheses were supported by the results from this study. Sense of coherence was associated with caregiver sense of competence and self-efficacy, which had not previously been studied in existing literature. Sense of coherence was strongly related to resilience, which was a unique finding with potentially important clinical implications. Previous literature has demonstrated an association between these variables but not to the same strength as this study [27]. However, this relationship warrants further investigation, given the strong association as it has not been well researched in caregivers of people living with dementia [28]. In addition, the correlation between sense of coherence to health-related quality of life demonstrates the importance of sense of coherence scale as a protective trait that may result in an improved health related 
quality of life for family caregivers, buffering against the challenges of being a caregiver. These findings reflect previous literature [11], which found that higher sense of coherence scores were associated with increased health related quality of life in caregivers.

The qualitative feedback demonstrated that caregivers felt that the items and scoring in the scale were not clear or needed updating as they were confusing, ambiguous, or offended the respondents, suggesting that the face validity could be improved. This may have arisen because the scale was developed for the general population.

\section{Methodological limitations of this research}

Despite the large sample size, it proved difficult to engage family caregivers from more diverse ethnic groups, particularly non-white caregivers. The online survey also proved more popular than the paper one, indicating the importance of providing different response options to participants. This may have targeted a more specific population, which could explain the higher number of adult-child caregivers than most studies of a similar population. In order to address these issues, effort was made to engage with black and minority ethnic groups and charitable organisations, but this did not significantly increase the ethnic diversity of respondents. Additionally, over half of the caregivers were not cohabiting with the person with dementia, which may have impacted findings as it could indicate that they were either not the primary caregiver or that they represent a distinct group of caregivers who were more likely to engage with a survey. This may reduce the generalisability of the results. However, there were no statistically significant differences between caregivers who cohabited and those that did not on any of the constructs included in this study.

Additionally, the inclusion criteria remained broad in order to include a wide variety of caregivers in the sample in order for the sample to be self-selecting. However, this may limit the generalizability of the results to validate the instrument to a specific population however it may increase the utility of the instrument to be used with all types of caregivers.

We were not able to assess the content validity in detail due to the design of this survey. Therefore further investigation into the content validity of this scale is recommended with this population, both by consulting a panel of experts and by asking family caregivers in depth about their opinion of the items included in the scale.

\section{Future research}

We recommend longitudinal or interventional research in order to investigate whether the SOC-13 is responsive to change and to evaluate test-re-test reliability in this population. Further guidance on potential modification should be considered, as responses from caregivers indicated some concerns about the face validity of the scale. Investigating this in a focus group would allow an in-depth examination into the face validity of the scale as a whole, and whether each item is suitable for use to measure sense of coherence in this population, leading to a potential revision of the scale.

In addition, findings from the CFA indicated that the factor structure of the SOC-13 was not confirmed to be a three-factor structure. Taken together with the qualitative findings, it indicates that the items and structure of the scale should be more comprehensively explored in terms of how well it measures sense of coherence, and the three subgroups within this domain (meaningfulness, comprehensibility and manageability). This could be explored in a focus groups design in order for caregivers to give an opinion as to how each of the questions reflect their own sense of coherence and measure how well they are able to find life meaningful, comprehensive and manageable.

\section{Conclusion}

This was the first study to investigate the psychometric properties of this scale in this population and in a self-report format. Overall, the scale had good reliability and the psychometric properties were good, indicating that the Sense of Coherence scale-13 could be a suitable instrument for use in clinical practice and interventional research. Additional guidance for caregivers completing the scale could help to improve the face validity.

\section{Abbreviations \\ CFA: Confirmatory Factor Analysis; JDR: Join Dementia Research; RMSEA: Root Mean Square Error of Approximation; RS-14: Resilience Scale - 14 items; SEMD: Self- Efficacy for Managing Dementia Scale; SOC-13: Sense of Coherence Scale - 13 items; SPSS: Statistical Package for the Social Sciences; SRMR: Standardised Square Root Mean Residual; SSCQ: Short Sense of Competence Questionnaire}

\section{Acknowledgements}

The VALID research team acknowledges the support of the National Institute for Health Research Clinical Research Network. The views expressed are those of the author(s) and not necessarily those of the NHS, the NIHR or the Department of Health.

The authors acknowledge with thanks the charitable organisations that assisted with recruitment for this study: Tapestry Havering, Age UK

Oxfordshire and Carers Oxfordshire.

\section{Funding}

This research was conducted as part of the Valuing Active Life in Dementia (VALID) research programme funded by the National Institute for Health Research (NIHR) under its Programme Grants for Applied Research scheme (RP-PG 0610-10108).

\section{Availability of data and materials}

The datasets used and/or analysed during the current study are available from the corresponding author on reasonable request. 


\section{Authors' contributions}

JS designed the survey, conducted the data collection, analysis and drafted the manuscript. JW was the second coder for thematic analysis of qualitative data. JW, MO \& MVD supervised and oversaw the conduct of the research and contributed to the manuscript. All authors read and approved the final manuscript.

\section{Ethics approval and consent to participate}

The investigation conforms to ethical principles. Ethical approval was granted by London-Camberwell St Giles NHS Research Ethics Committee REC Ref [14] LO/0736, 25.02.2016. Participation in this study was voluntary and participants were informed to be free to withdraw from the study at any time. The participants were provided with written information about the study process. Those who willingly agreed to participate in this study completed an online or paper consent form.

\section{Consent for publication}

Not applicable.

\section{Competing interests}

The authors declare that they have no competing interests.

\section{Publisher's Note}

Springer Nature remains neutral with regard to jurisdictional claims in published maps and institutional affiliations.

\section{Author details}

'Division of Psychiatry, University College London, Maple House, 149 Tottenham Court Road, London W1T 7BN, UK. ${ }^{2}$ Research and Development North East London NHS Foundation Trust, Goodmayes Hospital, Ifford IG3 8XJ, UK. ${ }^{3}$ Institute of Mental Health, University of Nottingham, Triumph Road, Nottingham NG7 2TU, UK. ${ }^{4}$ Radboud University Medical Centre, Postbus 9101, 6500 HB Nijmegen, The Netherlands.

Received: 8 February 2018 Accepted: 27 February 2019

Published online: 11 March 2019

\section{References}

1. World Health Organization. Dementia: a public health priority. World Health Organization; 2012. http://www.who.int/iris/handle/10665/75263.

2. Alzheimer's Society. Dementia 2014: Opportunity for change. London: Alzheimer's Society; 2014.

3. Cooper C, Balamurali TBS, Selwood A, Livingston G. A systematic review of intervention studies about anxiety in carers of people with dementia. Int $J$ Geriatr Psychiatry. 2007;22:181-8.

4. Crespo M, Lopez J, Zarit SH. Depression and anxiety in primary carers: a comparative study of carers of demented and nondemented older persons. Int J Geriatr Psychiatry. 2005;20:591-2.

5. Pinquart M, Sorensen S. Ethnic difference in stressors, resources and psychological outcomes of family caregiving: a meta-analysis. The Gerontologist. 2005;45:90-106.

6. Schulz R, Martire LM. Family caregiving of persons with dementia: prevalence, health effects, and support strategies. Am J Geriatr Psychiatry. 2004;12:240-9.

7. Vitaliano PP, Young HM, Zhang J. Is caregiving a risk factor for illness? Curr Dir Psychol Sci. 2004;13:13-6.

8. Antonovsky A. Health, stress, and coping. Michigan: Jossey-Bass; 1979.

9. Antonovsky A. Unraveling the mystery of health: how people manage stress and stay well. Michigan: Jossey-Bass; 1987.

10. Ekwall AK, Sivberg B, Hallbery IR. Older caregivers' coping strategies and sense of coherence in relation to quality of life. J Adv Nurs. 2007;57:584-96.

11. Andren S, Elmstahl S. Family caregivers' subjective experiences of satisfaction in dementia care: aspects of burden, subjective health and sense of coherence. Scand J Caring Sci. 2005;19:157-68.

12. Gallagher TG, Wagenfeld MO, Baro F, Haepers K. Sense of coherence, coping and caregiver role overload. Soc Sci Med. 1994;39:1615-22.

13. Antonovsky A. The structure and properties of the sense of coherence scale. Soc Sci Med. 1993;36:725-33.

14. Eriksson M, Lindström B. Validity of Antonovsky's sense of coherence scale: a systematic review. J Epidemiol Community Health. 2005;59:460-6.
15. Orgeta V, Sterzo EL. Sense of coherence, burden, and affective symptoms in family carers of people with dementia. Int Psychogeriatr. 2013;25(6):973-80.

16. Välimäki TH, Vehviläinen-Julkunen KM, Pietilä AM, Pirttilä TA. Caregiver depression is associated with a low sense of coherence and health-related quality of life. Aging Ment Health. 2009;13(6):799-807.

17. Wagnild G, Young H. Development and psychometric evaluation of the resilience scale. J Nurs Meas. 1993;1:165-78.

18. Wagnild G, Guinn P. The resilience scale user's guide for the U.S English version of the resilience scale and the 14-item resilience scale; 2011. p. 128.

19. Damásio BF, Borsa JC, da Silva J. P14-item resilience scale (RS- 14): psychometric properties of the Brazilian version. J Nurs Meas. 2011;19:13145 .

20. Vernooij-Dassen MJ, Felling AJ, Brummelkamp E, Dauzenberg MG, Bos GA, Grol R. Assessment of caregiver's competence in dealing with the burden of caregiving for a dementia patient: a short sense of competence questionnaire (SSCQ) suitable for clinical practice. J Am Geriatr Soc. 1999;47: 256-7.

21. Fortinsky RH, Kercher K, Burant CJ. Measurement and correlates of family caregiver self-efficacy for managing dementia. Aging Ment Health. 2002;6: $153-60$.

22. Eurogol Group. EuroQol-a new facility for the measurement of healthrelated quality of life. Health Policy. 1990;16:199-208.

23. IBM Corp. ReleasediBM SPSS Statistics for Macintosh, Version 25.0. Armonk, NY: IBM Corp; 2017

24. Braun V, Clarke V. Using thematic analysis in psychology. Qual Res Psychol. 2006:3:77-101

25. Jansen A, Hout H, Van Marwijk H, Nijpels G, Gundy C, Vernooij-Dassen M, De Vet $\mathrm{H}$, Schellevis F, Stalman W. Sense of competence questionnaire among informal caregivers of older adults with dementia symptoms: a psychometric evaluation. Clin Pract Epidemiol Ment Health. 2007;3:11.

26. Field A. Discovering statistics using SPSS. London: Sage Publications; 2009.

27. Trapp SK, Perrin PB, Aggarwal R, Peralta SV, Stolfi ME, Morelli E, Peña Obeso $L A$, Arango-Lasprilla JC. Personal strengths and health related quality of life in dementia caregivers from Latin America. Behav Neurol. 2015;2015:507196.

28. Cherry MG, Salmon P, Dickson JM, Powell D, Sikdar S, Ablett J. Factors influencing the resilience of carers of individuals with dementia. Rev Clin Gerontol. 2013;23(4):251-66.

Ready to submit your research? Choose BMC and benefit from:

- fast, convenient online submission

- thorough peer review by experienced researchers in your field

- rapid publication on acceptance

- support for research data, including large and complex data types

- gold Open Access which fosters wider collaboration and increased citations

- maximum visibility for your research: over $100 \mathrm{M}$ website views per year

At $\mathrm{BMC}$, research is always in progress.

Learn more biomedcentral.com/submissions 